\title{
Rendre le milieu hostile : contrôler et réprimer par le son, les gaz lacrymogènes et les projectiles «non-létaux »
}

\section{Christophe Wasinski}

\section{(2) OpenEdition Journals}

Édition électronique

URL : http://journals.openedition.org/conflits/22493

DOI : $10.4000 /$ conflits.22493

ISSN : $1777-5345$

Éditeur :

CECLS - Centre d'études sur les conflits - Liberté et sécurité, L'Harmattan

Édition imprimée

Date de publication : 15 décembre 2020

Pagination : 203-214

ISBN : 978-2-343-22588-3

ISSN : 1157-996X

Référence électronique

Christophe Wasinski, «Rendre le milieu hostile : contrôler et réprimer par le son, les gaz lacrymogènes et les projectiles « non-létaux » », Cultures \& Conflits [En ligne], 119-120 | automne-hiver 2020, mis en ligne le 15 février 2021, consulté le 01 avril 2021. URL : http://journals.openedition.org/conflits/22493 ; DOl : https://doi.org/10.4000/conflits.22493 
CHRONIQUE BIBLIOGRAPHIQUE 



\section{Rendre le milieu hostile : contrôler et réprimer par le son, les gaz lacrymogènes et les projectiles « non-létaux»}

\section{Christophe WASINSKI}

Christophe Wasinski est professeur en sciences politiques (relations internationales) à l'Université libre de Bruxelles. Il est membre du centre Recherche et Études en Politique Internationale (REPI). Ses recherches portent sur les questions militaires et de sécurité. Il a notamment publié dans les revues Actuel Marx, Critical Military Studies, Critique, Études Internationales, International Political Sociology et Security Dialogue.

Anna Feigenbaum, Petite histoire du gaz lacrymogène. Des tranchées de 1914 aux gilets jaunes, Paris, Libertalia, 2019.

Paul Rocher, Gazer, mutiler, soumettre. Politique de l'arme non létale, Paris, La Fabrique, 2020.

Juliette Volcler, Le son comme arme. Les usages policiers et militaires $d u$ son, Paris, La Découverte, 2011.

T es chercheurs qui se sont penchés sur la question des « désordres » ont mis

_en exergue l'existence de différentes normes dans le traitement de ceux-ci par les forces de sécurité. Des études telles que celle de Daniel Arasse sur la guillotine et son imaginaire politique, celle d'Eric Prokosh sur les armes antipersonnel de saturation de zone ou celle, plus récente, de Grégoire Chamayou sur les drones ont rappelé à quel point l'élimination physique a constitué et continue de constituer une modalité importante de la répression ${ }^{1}$. D’autres travaux, tels que l'ouvrage de Michel Foucault sur la prison, celui d'Olivier Razac sur les barbelés ou les analyses plus récentes de Laleh Khalili sur les

1. Arasse D., La guillotine et l'imaginaire de la terreur, Paris, Flammarion, 1987 ; Prokosh E., The Technology of Killing: A Military and Political History of Antipersonnel Weapons, Londres, Zed Books, 1995 ; Chamayou G., Théorie du drone, Paris, La Fabrique, 2013. 
opérations contre-insurrectionnelles menées au nom de la «guerre au terrorisme », insistent quant à eux sur l'enfermement comme manière de traiter et d'invisibiliser la contestation ${ }^{2}$.

Les ouvrages de Juliette Volcler et d'Anna Feigenbaum montrent qu'il existe également des techniques de contrôle et de répression des foules qui reposent sur la transformation du milieu. En l'occurrence, les deux auteures décrivent et analysent les étapes par lesquelles les gaz lacrymogènes et le son se sont imposés, au sein des services de sécurité, comme des moyens de rendre l'atmosphère hostile, momentanément toxique ou chargée de vibrations qui la rendent invivable, afin de contraindre les populations à se disperser et à réintégrer leurs domiciles. Ces équipements sont donc à l'origine d'un « maintien de l'ordre atmosphérique ». Le livre de Paul Rocher complète ce panorama tout en l'élargissant par la prise en considération des grenades et de lance-projectiles des forces de l'ordre.

Les équipements étudiés dans les trois livres sont par ailleurs généralement décrits comme des « armes non létales 3 ». Derrière ce terme rassurant se cachent des produits et des dispositifs utilisés pour réprimer avec brutalité. En certaines circonstances, ils peuvent même causer la mort. Même si elles ne sont pas conçues pour ôter la vie et faire couler le sang, comme l'écrit Steve Wright, " ces technologies sont faites pour avoir l'air et non pour être inoffensives ${ }^{4}$ ». Les deux chercheuses et le chercheur ont donc pour objet d'étude des armes de «férocité contrôlée » qui entretiennent des liens étroits avec l'imaginaire des « foules dangereuses 5 ». Enfin, l'intérêt des ouvrages de Juliette Volcler et d'Anna Feigenbaum réside aussi dans le fait qu'ils portent tous les deux sur les circulations militaro-policières des technologies de répression ${ }^{6}$.

2. Foucault M., Surveiller et punir. Naissance de la prison, Paris, Gallimard, 1975 ; Razac O., Histoire politique du barbelé, Paris, Flammarion, 2009; Khalili L., Times in the Shadows. Confinment in Counterinsurgencies, Stanford, Stanford University Press, 2013.

3. Ou «less than lethal» («moins que létales»). Lewer N., Schofield S., Non-lethal weapons: a fatal attraction? Military strategies and technologies for 21st-century conflict, Londres, Zed Books, 1997.

4. Wright S., "The New Technologies of Political Repression: A New Case for Arms Control”, Philosophy and Social Action, n ${ }^{\circ}$ 17, juillet-décembre 1991, cité (sans page) par Volcler J., Le son comme arme, op. cit., p. 139.

5. Rigouste M., La domination policière. Une violence industrielle, Paris, La Fabrique, 2013. L'auteur s'est aussi intéressé à cette catégorie d'armes, en particulier au pistolet à décharge électrique Taser. Sur l'imaginaire de la «foule dangereuse », voir : Razac O., Histoire politique du barbelé, op. cit. ; Potier E., "Imaginaire du contrôle des foules dans l'armée française », Cultures E Conflits, n56, 2004, pp. 35-49.

6. Bigo D., «La mondialisation de l'(in)sécurité ? Réflexions sur le champ des professionnels de la gestion des inquiétudes et analytique de la transnationalisation des processus d'(in)sécurisation », Cultures $\mathcal{E}$ Conflits, n58, 2005, pp. 53-101. 


\section{Les gaz lacrymogènes}

D'entrée de jeu, Anna Feigenbaum indique qu'elle a voulu faire le procès des gaz lacrymogènes dans son étude ${ }^{7}$. L'ouvrage commence par poser le problème à travers un tour du monde des usages de ces gaz. Y sont évoqués Bahreïn, le Brésil, le Chili, l'Égypte, les États-Unis, la France, Hong Kong, le Pakistan, la Turquie ou encore la Palestine. Le constat est accablant : la majorité des grands mouvements populaires de contestations récents ont fait, et font encore, l'objet de traitement par une consommation massive de gaz lacrymogènes, au Nord comme au Sud. Elle enchaîne par une rapide présentation des gaz - les agents lacrymatoires CS (2-chlorobenzylidène malonitrile), CN (chloroacétophénone), CR (dibenzoxazépine) et le gaz au poivre nommé OC (oléosine de capsicum). Entre autres choses, on apprend dans cette partie que ces agents ne provoquent pas que le larmoiement mais aussi des éruptions cutanées, des brûlures de la peau, des quintes de toux, des sensations d'asphyxie, des nausées ou encore des vomissements. Leur emploi est considéré comme dangereux dans les espaces clos. L'explosion de la grenade, qui permet la diffusion du gaz, peut également provoquer des blessures. Enfin, les gaz sont suspectés de provoquer des fausses couches et des maladies respiratoires chroniques.

Dans un second temps, Anna Feigenbaum retrace les étapes historiques qui ont mené à la normalisation du recours aux gaz lacrymogènes. Tout commence aux États-Unis à la fin de la Première Guerre mondiale. Lors de la guerre, les forces armées ont développé des capacités de recherche et de production en matière d'armement chimique. Après 1918, le Chemical Weapons Service (CWS) se recycle en menant des travaux sur les gaz lacrymogènes. Ces équipements sont notamment destinés à la Garde Nationale dont l'une des tâches est le maintien de l'ordre. Comme le souligne l'auteure, les militaires chargés de développer ces gaz sont par ailleurs proches des sociétés privées qui les produisent; on trouve parmi les cadres de ces sociétés d'anciens officiers. Au cours des années 1920, des discussions internationales abordent les questions relatives à l'interdiction de l'usage des gaz en général. Elles aboutissent à la signature du Protocole de Genève de 1925. Alors qu'il interdit aux militaires tout usage des gaz en situation de guerre, y compris donc les gaz lacrymogènes, il ne dit rien des usages domestiques de ces équipements ${ }^{8}$. Le CWS et l'industrie sauront exploiter cette opportunité.

7. Bien que le livre sur le son soit paru avant celui sur les gaz, nous commençons par présenter ce dernier. Les gaz lacrymogènes sont apparus avant les dispositifs répressifs sonores. En le présentant en premier, nous rendons mieux compte de l'évolution chronologique des techniques sécuritaires. Notons aussi que le livre d'Anna Feigenbaum est initialement paru en $2018 \mathrm{chez}$ Verso sous le titre : Tear Gas: From the Battlefield of World War I to the Streets of Today.

8. Voir aussi : Longuet S., «Les agents de lutte antiémeute, des armes chimiques hors du tabou ? ", Cultures E Conflits, n93, printemps 2014, pp. 163-168. 
De fait, selon Anna Feigenbaum, le général Amos Fries qui est à la tête du CWS - et qui est décrit comme conservateur, anticommuniste, xénophobe, suprémaciste blanc - travaille, pendant ces mêmes années 1920, à faire évoluer la perception que l'opinion a des gaz. Les journalistes, juristes et scientifiques impliqués dans cette campagne mettent en évidence le fait que les nouveaux gaz sont moins mortels que les balles. Leur discours repose sur l'argument selon lequel ces gaz sont des « armes humanitaires». Les fabricants de gaz démarchent les services de police des zones industrielles réputées tendues. Ils parviennent à éveiller l'intérêt des policiers. Les patrons voient également en ces équipements un moyen de lutter contre les grévistes.

En 1932, la Garde Nationale utilise du gaz pour réprimer brutalement la Bonus Army, un mouvement de protestation des vétérans de la Première Guerre mondiale qui réclament des primes en compensation de leurs sacrifices passés. Un enfant meurt asphyxié et une femme enceinte perd son bébé dans le contexte de cette répression menée par le général Douglas MacArthur. Des membres du Congrès se saisissent finalement de la problématique. L'innocuité de ces gaz est contestée lors de leurs enquêtes et la proximité entre le CWS et l'industrie est dénoncée.

L'expérience répressive états-unienne a entre-temps attiré l'attention des policiers et militaires dans les colonies britanniques. En 1919, plus de 370 personnes ont été tuées par balles à Amritsar, dans le Nord-Est des Indes, lors d'une manifestation contre des lois scélérates qui permettaient l'emprisonnement indéfini à titre préventif de toute personne suspectée de vouloir se rebeller (Rowlatt Act). Le développement d'un mouvement de désobéissance civile par Ghandi est l'occasion pour les autorités britanniques de se rendre compte qu'il leur faut trouver de nouvelles solutions pour gérer la contestation politique. Ces autorités cherchent alors à se faire livrer des gaz - des représentants des fabricants états-uniens prennent acte de cet intérêt et commencent à sillonner les colonies. La domination coloniale est « modernisée » par l'emploi de ces armes. Anne Feigenbaum note qu'en Europe également, des gaz lacrymogènes sont utilisés pour réprimer des mouvements sociaux.

Anna Feigenbaum souligne encore la dimension transnationale de sa problématique lorsqu'elle indique que les Britanniques prennent le relais des États-Uniens en matière de recherche sur les gaz. La question du stockage est l'une des plus importantes pour les Britanniques car les gaz lacrymogènes résistent assez mal au climat tropical que l'on rencontre dans de nombreuses régions de l'empire. Les Britanniques expérimentent ensuite des gaz qui périment moins rapidement et qui sont plus puissants (le gaz lacrymogène CS puis ensuite le CR). Pendant les années 1960, les grenades qui contiennent ces gaz sont vendues à de nombreux États du Sud comme Hong Kong, le Kenya, le Nigéria, la Rhodésie, Singapour ou encore l'Indonésie). On les utilise égale- 
ment dans les États les plus industrialisés. Les forces de police des États-Unis en acquièrent aussi et les tirent dans les grandes villes pour réprimer des manifestations.

Le Royaume-Uni va, quant à lui, recourir à ces grenades lacrymogènes en Irlande du Nord, c'est-à-dire sur son propre territoire. L'opinion finit par s'émouvoir de l'usage massif que les forces militaires et de police en font en août 1969 lors du soulèvement du Bogside, le quartier populaire et catholique de Derry ${ }^{9}$. Il est vrai que l'usage de gaz dans les colonies, au Vietnam et aux États-Unis à la même époque avaient déjà généré des inquiétudes et des débats, notamment au Royaume-Uni ${ }^{10}$. C'est dans ce contexte qu'est formée la commission d'enquête Himsworth. Ses conclusions, qui sont publiées dans le Report of the enquiry into the Medical and Toxicological aspects of CS, sont contestées ${ }^{11}$. On reproche à la commission de s'être trop appuyée sur l'expertise des militaires pour rédiger le document. Quoi qu'il en soit, les gaz lacrymogènes sont encore présentés comme une « arme humanitaire » dans ce rapport. Certains experts considèrent même à l'époque que cet équipement serait finalement plus proche du médicament que de l'arme. Les effets secondaires restent pourtant mal connus, de même que les conséquences pour les enfants, les personnes âgées ou encore les femmes enceintes.

Les gaz continueront donc à être utilisés et les conclusions du rapport Himsworth resurgiront ponctuellement pour attester de leur innocuité. Elles sont par exemple évoquées en 1989 lorsque le Département d'État décide de valider la vente à Israël de gaz pour une valeur de 6,5 millions de dollars. Elles sont aussi citées lors de l'affaire de Waco au Texas. Les forces de sécurité emploieront notamment du gaz dans le brutal assaut contre les bâtiments de la secte de David Koreh. Autrement dit, comme le montre Anna Feigenbaum, le discours de justification de l'usage des gaz circule, comme les gaz eux-mêmes, au niveau transnational.

L'étude d'Anna Feigenbaum décrit brillamment le processus par lequel une conception offensive du maintien de l'ordre, découlant de l'usage des gaz lacrymogènes, s'est imposée. Elle montre clairement aussi ce que cette « arme humanitaire » doit aux militaires. À la lecture de son ouvrage, on songe aussi aux travaux d'Hannah Arendt sur l'impérialisme ${ }^{12}$. La philosophe avait mon-

9. La bataille du Bogside et la répression policière massive qui s'ensuivit contribueront à la réhabilitation de l'IRA qui s'était fourvoyée avec le nazisme. C'est suite à ces événements que se forme la Provisional Irish Republican Army.

10. Sur l'usage des armes chimiques au Vietnam, voir par exemple: Hersh S.M., Chemical and Biological Warfare, Londres, MacGibbon \& Kee,1968, pp. 53-62 et pp. 144-187.

11. Feigenbaum A., Petite histoire du gaz lacrymogène. Des tranchées de 1914 aux gilets jaunes, Paris, Libertalia, 2019, p. 206. Voir également : Balmer, B., Spelling, A., McLeish, C., "Tear Gas Epistemology: The Himsworth Committee and Weapons as Drugs", in Mankoo A., Rappert B. (eds.), Chemical Bodies: The Techno-Politics of Control, Lanham, Rowman \& Littlefield, pp. 77-102. 
tré comment les colonies avaient servi de terrain d'expérimentation de diverses techniques coercitives qui, dans un second temps, par « effet boomerang ", sont revenues dans les métropoles. Les gaz lacrymogènes ont également, en partie, suivi ce cheminement. Sans conteste, le livre, même s'il n'épuise pas les questions que l'on peut se poser sur le développement et l'usage des gaz lacrymogènes, apporte des éléments de compréhension précieux ${ }^{13}$.

\section{Le son comme arme}

L'étude de Juliette Volcler sur le son éclaire également la problématique $\mathrm{du}$ recours aux armes dites non létales et $\mathrm{du}$ « maintien de l'ordre atmosphérique ». Comme Anne Feigenbaum, elle commence par dépeindre les effets du son diffusé à très haut volume sur les personnes. On apprend donc qu'un son dont l'intensité est située entre 140 et $170 \mathrm{~dB}$ va provoquer des difficultés respiratoires, une sensation d'oppression à la poitrine, une salivation accrue, une vision floue, des fourmillements, des vertiges, des acouphènes, des maux de têtes, de la fatigue et une accélération cardiaque. À $160 \mathrm{~dB}$, les tympans risquent de se déchirer. À $200 \mathrm{~dB}$, les poumons peuvent se fissurer. Enfin, à 210 $\mathrm{dB}$, des hémorragie internes peuvent survenir. À ce volume, le son peut s'avérer mortel. Bref, des systèmes ou grenades capables de produire ces sons doivent être considérés comme des armes.

L'ouvrage montre ensuite qu'il existe de longue date une réflexion sur les « armes soniques ». Dans cette partie, Juliette Volcler évoque quelques idées fantaisistes développées par des chercheurs depuis des décennies et passe en revue des projets militaires n'ayant pas abouti - il s'agit notamment de programmes qui portaient sur la militarisation des infra-sons ${ }^{14}$. Ces idées et projets révèlent d'emblée l'existence d'un imaginaire de la « nuisance » autour du son. Celui-ci s'est par ailleurs concrétisé par l'usage des haut-parleurs sur les champs de bataille. Ces haut-parleurs sont des outils de guerre psychologique. On peut les utiliser pour diffuser des messages décourageants ou des sons qui agacent, qui épuisent, qui nuisent. Ainsi, lors de la guerre du Vietnam, des haut-parleurs montés sur des hélicoptères états-uniens diffusaient la "Wandering Soul », une bande composée de sons issus des films d'horreurs, d'enregistrement d'enfants qui pleurent et de musiques de funérailles bouddhistes. Pendant l'intervention des États-Unis à Panama en 1989-1990, les militaires diffusèrent vingt-quatre heures sur vingt-quatre, par haut-parleurs, de la musique beavy metal afin de faire sortir Manuel Noriega de la noncia-

12. Arendt H., L'impérialisme. L'origine du totalitarisme, Fayard, Paris, 1982.

13. Comme l'explique l'auteure en début d'ouvrage, l'analyse porte principalement sur les cas des États-Unis et de la Grande-Bretagne. L'Allemagne, la France ou encore l'Afrique du Sud devraient, selon Anna Feigenbaum, encore faire l'objet d'études systématiques.

14. Volcler J., Le son comme arme. Les usages policiers et militaires du son, Paris, La Découverte, 2011, pp. 27-48. 
ture au sein de laquelle il s'était réfugié. Les pilotes israéliens, quant à eux, passent le mur du son à basse altitude au-dessus des territoires occupés ou, à l'époque, au Liban afin de provoquer des «bangs » à répétition. Il s'agit ici d'un usage punitif du son qui vise une population. Des enfants palestiniens de moins de 16 ans sont victimes de crises d'angoisse, d'incontinence urinaire, de spasmes musculaires ou encore de difficultés respiratoires ${ }^{15}$. Les vitres de bâtiments peuvent aussi se fissurer du fait de ces vols. Les militaires considèrent quant à eux que leurs actions n'étant pas mortelles, elles peuvent être considérées comme « humaines ».

Dans ce panorama, il faut aussi présenter les haut-parleurs surpuissants tels que le Long Range Acoustic Device (LRAD) ${ }^{16}$. Le LRAD a été utilisé par les militaires états-uniens en Irak à Falloujah. Il a aussi été déployé par les forces de police des États-Unis à diverses occasions, notamment en NouvelleOrléans après le passage de l'ouragan Katrina. La police japonaise l'a quant à elle utilisé contre des activistes écologistes. La police canadienne l'a aussi déployé lors du sommet du G-20 de Toronto. Acheté par les forces de police d'une vingtaine d'États, le dispositif a été monté sur des navires militaires ou de gardes côtes. En 2005, monté sur un navire de croisière, il est utilisé contre des pirates somaliens ${ }^{17}$. Selon les situations, les autorités peuvent s'en servir comme un moyen de communication ou comme une arme. L'armée israélienne disposerait aussi d'un système de ce type nommé "the Shout ", "the Shriek » ou encore "the Scream». C'est également dans cette catégorie que l'on placera le «mosquito 18 ». Conçu en Grande-Bretagne par Compound Security Systems, ce boîtier émet des sons désagréables de manière à chasser des individus d'une zone donnée. C’est un équipement de lutte contre les « comportements asociaux ». L'appareil peut même produire des sons entendus uniquement des adolescents, leurs oreilles étant plus sensibles que celles des adultes.

Les détonations provoquées par des explosions peuvent également être utilisées dans un but répressif, comme le montre bien Juliette Volcler. Le " canon effaroucheur », dont on se sert pour éloigner les oiseaux des champs, a servi de modèle en la matière. On trouve une illustration de son usage militaro-policier avec les systèmes israéliens nommés «Thunder Generator » ou «Shockwave 19 ». Ceux-ci sont capables de produire des «bangs » puissants à répétition qui provoquent des douleurs aux oreilles. Comme Juliette Volcler le note, il faut aussi prendre en considération les grenades qui provoquent la

15. Ibid., p. 62.

16. Lire aussi : Bricet des Vallons G.-H, «L’arme non létale dans la stratégie militaire des ÉtatsUnis : imaginaire stratégique et genèse de l'armement », Cultures E Conflits, n67, 2007, pp. 63-82.

17. Volcler J., Le son comme arme, op. cit., p. 117.

18. Ibid., pp. 123-126.

19. Ibid., p. 59. 
« saturation sensorielle ». Afin d'entraîner les militaires à leurs usages, les fabricants de grenades ont inventé des modèles spéciaux d'exercice. Ces modèles ont la particularité d'exploser en ne provoquant pas (ou peu) d'éclats mais en générant un bruit douloureux. Les grenades dont les policiers se servent depuis des décennies lors des manifestations dupliquent en fait ces modèles d'entraînement. Une grenade états-unienne M-84 provoque par exemple un bruit d'une puissance de 170 à $180 \mathrm{~dB}$ à une distance d'un mètre et demi. Les fabricants ont aussi conçu des grenades pouvant provoquer deux, six, sept, voire neuf « bangs ». Ces grenades sont aussi utilisées par l'administration pénitentiaire aux États-Unis. Depuis 2004, alors que Nicolas Sarkozy est au ministère de l'Intérieur, les policiers français reçoivent des grenades de ce type. Elles sont nommées DMP (Dispositif Manuel de Protection). Selon les descriptions qui en sont données, leur effet est comparable à un fort coup de poing ou une gifle. Une fois de plus, l'auteure expose bien, par cet exemple, les transferts de techniques entre le champ militaire et le champ policier ${ }^{20}$.

L'auteure explique enfin que l'absence totale de son peut également produire des souffrances. C'est d'ailleurs la raison pour laquelle on y recourt dans le cadre des techniques de torture basées sur la privation sensorielle ${ }^{21}$. Notons néanmoins que, dans les faits, les forces de sécurité mélangent régulièrement privation de son et diffusion à fort volume d'enregistrements de bruit et de musique dans le but de « désorienter » et «briser » les détenus (musique rock associée à la « rage masculine 22 »; bruit amplifié d'une goutte d'eau qui tombe sur l'étain ; diffusion en boucle des cris enregistrés du détenu ou de pleurs de bébés). Ces techniques ont fait l'objet d'une codification dans des manuels qui ont ensuite servi à des enseignements dispensés pendant les années 1960 et

20. On notera qu'il existe une littérature assez abondante sur la militarisation du maintien de l'ordre. Cette littérature tend à se focaliser sur le recours aux forces armées dans les tâches policières. Il existe aussi une littérature qui met en exergue la « policiarisation » de certaines missions militaires. Voir par exemple : Bigo D., Guittet E.-P., « Militaires et sécurité intérieure. L’Irlande du Nord comme métaphore », Cultures $\mathcal{E}$ Conflits, n 54 , 2004, pp. 510 ; Rigouste M., L'ennemi intérieur. La généalogie coloniale et militaire de l'ordre sécuritaire dans la France contemporaine, Paris, La Découverte, 2011 ; Neocleous M., War Power, Police power, Edinburgh, Edinburgh University Press, 2014 ; Wood L. J., Mater la mente. La militarisation de la gestion policière des manifestations, Montréal, Lux, 2015 ; Bachmann J., Bell C., Holmqvist C., War, Police and Assemblages of Intervention, Londres, Routledge, 2015 ; Flores-Macías G.A., Zarkin J., "The Militarization of Law Enforcement: Evidence from Latin America”, Perspective on Politics, vol. 18, n¹, décembre 2019, pp. 1059-1078. En revanche, les analyses qui se concentrent sur les transferts d'équipements et d'armements entre les deux champs sont plus rares. Pour une réflexion générale à ce propos, lire : Dupont B., « Technologie, défense nationale et sécurité intérieure : un ménage à trois dysfonctionnel », in Lemieux F., Dupont B., La militarisation des appareils policiers, Québec, Les Presses de l'Université Laval, 2005, pp. 135-156.

21. Voir également : Kubark, Le manuel secret de manipulation mentale et de torture psychologique de la CIA, Paris, Zones, 2012.

22. Pour reprendre d'expression de la musicologue Suzanne Cusick, "Music as torture / Music as weapon”, Trans, n¹0, 2006 (consulté le 10 décembre 2019 sur : www.sibetrans.com/trans/articulo/152/music-as-torture-music-as-weapon), citée par Volcler J., Le son comme arme, op. cit., p. 93. 
1970 au sein de l'Office of Public Safety, aux États-Unis, à des policiers issus de 47 États. Des expérimentations en matière de privation sensorielle ont également eu lieu en Allemagne à la même époque sur des cobayes volontaires, notamment des soldats. Ces techniques seront appliquées en prison sur les membres de la Rote Armee Fraktion (RAF). La Suède et les Pays-Bas s'y intéresseront également. Les Portugais développeront leurs propres méthodes dans les colonies. La Grande-Bretagne n'est pas non plus en reste. Les forces de sécurité de cet État s'intéressent également à la privation sensorielle dans le contexte de la répression coloniale. Les techniques les plus physiques utilisées dans leur empire ayant été décriées, comme le soulignait aussi Anna Feigenbaum dans son étude sur les gaz lacrymogènes, les Britanniques cherchent à développer de nouvelles méthodes, dont celles reposant sur la privation sensorielle. Elles seront par exemple appliquées en Irlande du Nord. Les États-Unis les utiliseront également contre des détenus dans le contexte de la "guerre contre le terrorisme».

L'étude de Juliette Volcler est moins historique que celle d'Anna Feigenbaum. Son point de vue est également davantage technique. Elle décrit plus longuement les spécificités des nombreux équipements évoqués. La question coloniale n'est pas vraiment abordée dans son ouvrage. En revanche, elle met en exergue les points de contact entre les univers militaires et policiers des armes dites non-létales. Elle montre aussi à quel point ces techniques se sont nourries de recherches menées par l'industrie musicale et cinématographique. Son étude nous permet de mieux comprendre comment une conception du contrôle et de la répression des populations basée sur la transformation de l'atmosphère, par saturation de vibrations douloureuses, s'est développée ces dernières décennies.

\section{Les armes « non-létales » et l'écosystème répressif}

L'ouvrage de Paul Rocher est en prise directe avec la contestation sociale récente. Il porte non seulement sur l'usage des gaz de dispersion de foules mais également sur les grenades des forces de l'ordre et lanceurs de projectiles. Son questionnement, inspiré par celui de Grégoire Chamayou et de Simone Weil, consiste à partir des moyens pour remonter vers la détermination des fins politiques. L'auteur cherche à comprendre l'effet que les équipements non létaux ont sur les forces de sécurité et, par contrecoup, sur les manifestants.

L'ouvrage s'intéresse pour commencer à l'usage de gaz. Le propos recoupe celui d'Anna Feigenbaum, qui est citée par l'auteur. Il insiste cependant davantage sur l'usage de ces gaz en France, notamment au cours des années 1960. Pour Paul Rocher, les CRS et les policiers étaient alors d'autant plus enclins à user de la force pour réprimer les manifestations qu'ils considéraient les juges trop timorés pour les punir. L'auteur aborde ensuite l'usage des canons à eau et de l'électricité. Associée à la torture, cette dernière néces- 
site un «rebranding » pour pouvoir être vendue aux forces de l'ordre sous forme du Taser. Paul Rocher mentionne aussi les matraques avant de passer aux balles en caoutchouc utilisées par les forces de sécurité en Irlande du Nord ou dans les territoires occupés lors de la première Intifada. Vient ensuite une partie qui se focalise sur les lanceurs de projectiles Flash Ball puis LBD 40. Enfin, il est question des grenades de désenclavement, telle que la GLI-F4, produisant des bruits douloureux et des éclats qui blessent.

L'introduction de cette panoplie dans l'arsenal des forces de l'ordre a plusieurs conséquences. Tout d'abord, de façon générale, dotés d'équipement, les policiers peuvent atteindre les manifestants à plus grande distance. Leur matériel est clairement devenu plus « performant ». Ensuite, « la disponibilité des armes non létales conduit les forces de l'ordre à frapper, à gazer et à tirer davantage 23 ». Il est donc question d'abaissement du seuil du recours à la force du fait de la mise à disposition de ces armes. C'en est à un tel point que l'auteur en vient à se demander s'il ne faudrait pas considérer, à l'instar de Gary T. Marx, qu'il y a par moment des «émeutes de policiers » face aux manifestants ${ }^{24}$. À la lueur de ces éléments, l'humanisation du maintien de l'ordre apparaît comme une illusion - sans compter que l'usage des armes classiques est resté constant, les armes dites non-létales ne se substituant pas à celles-là. On pourrait même plutôt parler de brutalisation de l'action policière. Ce terme s'avère d'autant plus pertinent si l'on considère que la pression des jets d'eau a quasiment doublé depuis l'entrée en service de ceux-ci, que les distances de sécurité recommandées pour le lancement des projectiles se sont amenuisées ou encore que la puissance du Taser a été augmentée. L'auteur évoque aussi deux formes de «panachage » technique avec le mélange des gaz à l'eau sous pression par les forces turques à Gezi en 2013 et l'introduction de restes nauséabonds d'animaux morts aux jets des autopompes par les policiers français. L'auteur rappelle par ailleurs qu'il est rare que les utilisateurs de ce type d'équipements s'efforcent d'étudier leur dangerosité réelle ${ }^{25}$.

L'ouvrage s'intéresse ensuite à ceux qui utilisent ces équipements, c'est-àdire les membres des forces de l'ordre ${ }^{26}$. Le chapitre qui porte sur ceux-ci analyse les profils et l'esprit de corps des policiers. Paul Rocher rappelle au passage que la majorité des membres des forces de l'ordre qui ont été déployés dans des opérations contre des manifestants ont fait l'expérience de la peur face à la foule, ce qui n'est pas sans lien avec leur propension à user des armes.

23. Rocher P., Gazer, mutiler, soumettre. Politique de l'arme non létale, Paris, La Fabrique, 2020, p. 16.

24. Marx G. T., "Civil Disorder and the Agents of Social Control”, Journal of Social Issues, vol. $26, \mathrm{n}^{\circ} 1,1970$, pp. 19-57.

25. Voir aussi : Samuel A., Picot A. et al., L'utilisation du gaz lacrymogène CS : ses effets à long terme, Association Toxicologie-Chimie de Paris, juillet 2020 (version en cours de relecture, http://www.atctoxicologie.fr/images/Gaz_lacrymo_CS_DossierV7.pdf).

26. À ce propos, voir aussi : Fassin D., La force de l'ordre. Une anthropologie de la police des quartiers, Paris, Seuil, 2011. 
Plus loin dans l'ouvrage, il souligne aussi qu'en certaines circonstances la police s'est sentie débordée par les protestations. Enfin, Paul Rocher enchâ̂ne avec un chapitre sur «l'autodéfense populaire » dans lequel il étudie le comportement des manifestants qui sont visés par les armes dites non-létales. Il note qu'ils ne sont pas passifs mais tentent de s'organiser et de développer des contre-mesures. Ainsi, les manifestants emportent des boucliers, des boules Quies, des lunettes et des masques de plongée et ils imbibent leurs foulards de citron ou de menthe afin d'atténuer les effets des gaz. La répression s'adapte elle-même et, lors des contrôles, les policiers saisissent ce qui permet aux manifestants de se protéger. L'action des forces de l'ordre a donc aussi pour objectif d'assurer la vulnérabilité de ceux qui protestent. Les manifestants tentent également de politiser les effets des armes sur leurs corps, comme en atteste une marche des Gilets jaunes blessés à Paris en février 2019.

Le rôle de l'industrie de la sécurité est aussi abordé. L'auteur rappelle qu’à Notre-Dame-des-Landes en 2018, les gendarmes ont tiré en huit jours pas moins de 11000 grenades de différents types, soit environ 1400 grenades par jour. Pour le dire autrement, avec de telles consommations, les armes nonlétales constituent un marché alléchant pour les fabricants de matériel. Mais il y a plus. Ces équipements connaissant beaucoup de succès à l'exportation, et pas que vers les États les plus démocratiques. L'Afrique du Sud, l'Arabie saoudite, Bahreïn, le Burkina Faso, l'Égypte ou encore la Tunisie sous Ben Ali se sont par exemple montrés très intéressés par le savoir-répressif français et ont acquis ces équipements au cours des dernières années ${ }^{27}$.

Enfin, l'ouvrage cherche à montrer comment les évolutions du maintien de l'ordre, rendues notamment possibles par l'introduction des armes dites non-létales, sont en phase avec le projet économique néolibéral qui impose des mesures d'austérité aux populations. Dans ce sillage, Paul Rocher revient rapidement sur les travaux des grands penseurs classiques de la société et de l'État, tels que Nicos Poulantzas, Pierre Bourdieu ou Michel Foucault. Ces derniers avaient insisté sur le consentement social obtenu grâce à un pouvoir aux effets subtils et aux discrètes manipulations idéologiques. D'après Paul Rocher, le durcissement des politiques sécuritaires invite à se montrer prudent dans l'analyse et à ne pas trop rapidement perdre de vue le rôle joué par la force physique. Sans aucun doute, cette remarque vaut aussi pour les livres de Juliette Volcler et d'Anna Feigenbaum.

Comme en atteste l'actualité récente, le recours aux armes dites non létales par les forces de sécurité à travers le monde est tout sauf anecdotique. Dans un

27. Notons au passage que Pascal Menoret, dans son étude sur la jeunesse en Arabie saoudite, observe que cet État a été conçu pour la voiture. Les espaces propices à la socialisation et donc aussi à la contestation sont quasi-inexistants. De fait, l'urbanisme, sans être véritablement une arme, y facilite le contrôle policier et politique. Menoret P., Royaume d'asphalte. Jeunesse saoudienne en révolte, Paris, La Découverte, 2016. 
rapport datant de 2018, le Haut-Commissariat des Nations unies aux droits de l'homme s'inquiétait de la situation au Cachemire. Le rapport dénonçait entre autres l'usage que les forces indiennes faisaient de la chevrotine («pellets ») afin de contrôler les foules ${ }^{28}$. Lors des manifestations au Chili à l'automne 2019, les forces de l'ordre, accusées de faire un usage excessif de la violence, ont notamment utilisé des gaz lacrymogènes, des balles de caoutchouc et de la chevrotine ${ }^{29}$. Durant l'été 2020, des manifestations se sont déroulées dans de nombreux États à travers le monde. En Bélarus, en Côte-d'Ivoire, aux États-Unis, en Guinée, au Liban, en Tunisie et au Venezuela, les policiers ont également fait un usage excessif de la force pour réprimer les protestataires selon Amnesty International 30. L'organisation remarquait que, dans tous ces États, les forces de l'ordre avait eu recours à des armes dites non létales. Au mois de septembre 2020, les forces de sécurité camerounaises utilisaient des canons à eau et gaz afin de réprimer des manifestations ${ }^{31}$. Un mois plus tard, Human Rights Watch critiquait l'emploi de canons à eau et des gaz par la police thailandaise contre des manifestants pacifiques qui s'opposaient au régime en place ${ }^{32}$. Toujours au mois d'octobre 2020, les forces de sécurité nigérianes recouraient notamment au gaz lacrymogène et aux canons à eau contre les manifestants qui s’étaient rassemblés pour dénoncer des brutalités policières ${ }^{33}$. Ces exemples récents, qui illustrent l'ampleur du phénomène, prouvent qu'une réflexion de fond sur ces équipements et leurs usages s'avère urgente. Les ouvrages d'Anna Feigenbaum, de Juliette Volcler et de Paul Rocher ont ouvert une voie critique qui peut s'avérer féconde dans ce domaine.

28. Office of the United Nations High Commissioner for Human Rights, Update of the Situation of Human Rights in Indian-Administered Kashmir and Pakistan-Administered Kashmir from May 2018 to April 2019, 8 juillet 2019, pp. 16-17, https://www.ohchr.org/Documents/Countries/IN/KashmirUpdateReport_8July2019.pdf. Consulté le 13 décembre 2020.

29. Report of the Mission to Chile 30 October - 22 November 2019, United Nations Human Rights, décembre 2019, p. https://www.ohchr.org/Documents/Countries/CL/Report_Chile_2019_EN.pdf ; Eyes on Chili: Police Violence and Command Responsability during Period of Social Unrest, Amnesty International, octobre 2020, https://www.amnesty.org/download/Documents/AMR2231822020ENGLISH.PDF. Consulté le 13 décembre 2020.

30. Castner B., "Police Riot Gear, Old and New", Amnesty International, 8 septembre 2020, https://citizenevidence.org/2020/09/08/police-riot-gear-old-and-new/ Consulté le 13 décembre 2020.

31. "Cameroon: Opposition Leaders, Supporters Detained Release Those Held Arbitrarily; End Crackdown on Dissent", Human Rights Watch, 19 octobre 2020, https://www.hrw.org/news/2020/10/19/cameroon-opposition-leaders-supporters-detained Consulté le 13 décembre 2020.

32. "Thailand: Water Cannon Used Against Peaceful Activists Protesters, Journalist Arrested Under Draconian Emergency Decree", Human Rights Watch, 17 octobre 2020, https://www.hrw.org/news/2020/10/17/thailand-water-cannon-used-against-peacefulactivists Consulté le 13 décembre 2020.

33. "Nigeria: Crackdown on Police Brutality Protests Prosecute Officers Responsible; Move Toward Systemic Reform", Human Rights Watch, 16 octobre 2020, https://www.hrw.org/news/2020/10/16/nigeria-crackdown-police-brutality-protests Consulté le 13 décembre 2020. 